\title{
TRIPLET ENERGY MIGRATION IN POLYMER LANGMUIR-BLODGETT FILMS
}

\author{
KENI HISADA, HIDEAKI KATAYAMA, SHINZABURo ITO, and MASAHIDE YAMAMOTO \\ Department of Polymer Chemistry, Faculty of Engineering, \\ Kyoto University, Sakyo-ku, Kyoto 606 JAPAN
}

The excited triplet state of carbazole chromophores in Langmuir-Blodgett (LB) films for carbazole containing polymers, i.e., copolymers $(\mathrm{P}(\mathrm{C}-\mathrm{iB}))$ of 2-(9carbazolyl)ethyl methacrylate (CzEMA) with isobutyl methacrylate (iBMA) and a copolymer (P(C-OD)) of CzEMA with octadecyl methacrylate (ODMA), was investigated. In the case of $\mathrm{P}(\mathrm{C}-\mathrm{iB})$, an interlayer interaction plays a role when more than four layers of chromophoric layers are deposited. It was confirmed that triplet energy migration between triplet chromophores mainly depends on the interchromophore distance, whether the system consists of 2-dimensional LB film or 3-dimensional amorphous polymer film.

\section{Introduction}

Electronically excited states of aromatic chromophores in polymer systems have been widely investigated [1]. In a condensed phase of polymer matrices, various interchromophoric processes, such as energy migration and excimer formation usually take place and elucidation of the mechanisms is important from the standpoint of photofunctional polymers. In previous works [2-6], we have investigated the behavior of the excited triplet states of aromatic chromophores in polymer matrices by phosphorescence spectroscopy.

We studied the triplet energy migration and the formation of triplet excimers in polymer Langmuir-Blodgett (LB) film containing carbazole moieties, and compared them with the behavior 
in the film prepared by a conventional cast method.

\section{Experimental}

Materials. The chromophoric copolymers were prepared by copolymerization of 2-(9-carbazolyl)ethyl methacrylate (CzEMA) with isobutyl methacrylate or octadecyl methacrylate using azobisisobutyronitrile as an initiator. These copolymers are called $\mathrm{P}(\mathrm{C}-\mathrm{iB})$ and $\mathrm{P}(\mathrm{C}-\mathrm{OD})$, respectively. The obtained copolymers were purified by repeated reprecipitation from benzene to methanol and dried in vacuo. Octadecyl methacrylate (Tokyo Kasei Kogyo Co. Ltd.) was purified by recrystallization from acetone. Poly(vinyl octanal acetal) (PVO) was synthesized by acetalization of poly(vinyl alcohol) with octanal. Further details for preparing $\mathrm{P}(\mathrm{C}-\mathrm{iB})$ and $\mathrm{PVO}$ were described elsewhere [7-9]. Table I shows the characteristics of the polymers. PVO was used as spacer layers between the chromophoric layers. PVO was also used as precoating layers and surface protection layers to avoid the effect of the interface.

Sample Preparation.The $0.1 \mathrm{~g} / 1$ benzene solution of each copolymer was spread on pure water at $19^{\circ} \mathrm{C}$. The monolayer on the water surface was transferred onto a quartz plate by the lifting up and down method at an appropriate surface pressure $\left(\pi_{\mathrm{D}}\right)$. The surface of quartz plate was cleaned in oxidative sulfuric acid, and rinsed repeatedly with water. To make the surface hydrophobic, the plate was dipped in a 10\% trimethylchlorosilane solution in toluene for $30 \mathrm{~min}$. $\mathrm{P}(\mathrm{C}-\mathrm{iB})$ was transferred on a hydrophobic substrate and $\mathrm{P}(\mathrm{C}-\mathrm{OD})$ was transferred on a hydrophilic substrate. The dipping speed was maintained at $15 \mathrm{~mm} / \mathrm{min}$ for $P(C-i B)$, and at $15 \mathrm{~mm} / \mathrm{min}$ (upward) and $2 \mathrm{~mm} / \mathrm{min}$ (downward) for P(C-OD). Under these conditions, the transfer ratio was nearly unity and the transfer mode was in $\mathrm{Y}$-type for $\mathrm{P}(\mathrm{C}-\mathrm{iB})$ and in $\mathrm{Z}$-type for $\mathrm{P}(\mathrm{C}-\mathrm{OD})$. The P(C-iB) LB film without spacer layers has the following layer sequence : (1) 4 layers of PVO

Table I Composition of copolymers, average interchromophore distance, and surface pressure at deposition.

\begin{tabular}{lccc}
\hline \multicolumn{1}{c}{ Code } & $\begin{array}{c}\text { CzEMA } \\
\text { mol\% }\end{array}$ & $\begin{array}{l}\mathrm{R}_{2 \mathrm{D}} \\
\mathrm{nm}\end{array}$ & $\begin{array}{c}\pi_{\mathrm{D}} \\
\mathrm{mN} / \mathrm{m}\end{array}$ \\
\hline P(C-iB)5 & 5.6 & 1.98 & 9 \\
P(C-iB)10 & 9.8 & 1.49 & 8.5 \\
P(C-iB)15 & 16.3 & 1.32 & 6 \\
P(C-OD) & 15.7 & 1.13 & 20 \\
PODMA & 0 & - & 20 \\
PVO & - & - & 20 \\
\hline
\end{tabular}


(a)

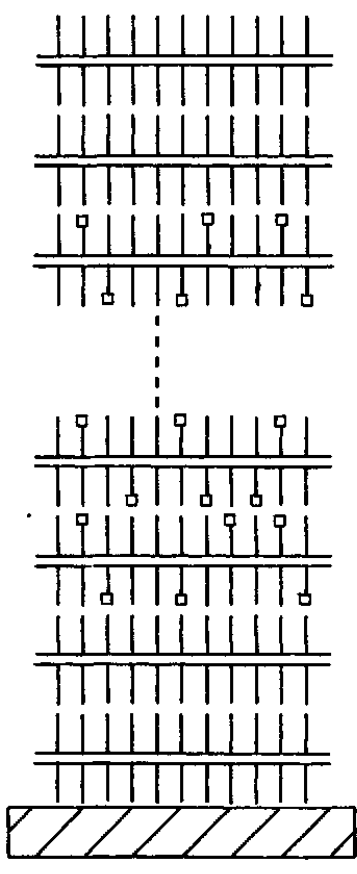

(b)

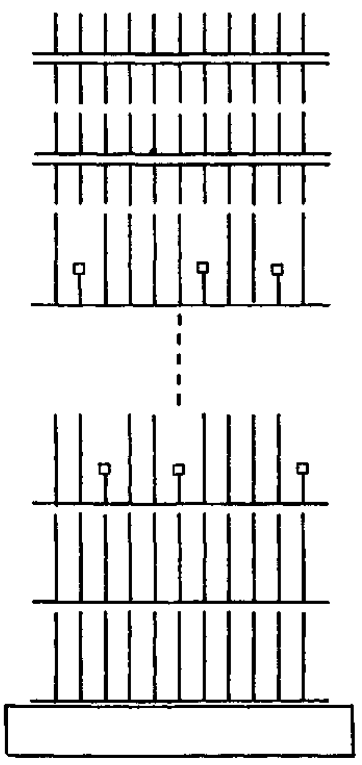

(c)

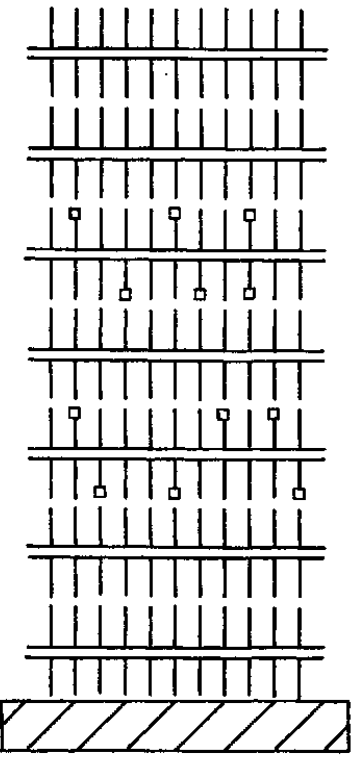

Fig. 1 Schematic illustration of LB films. Squares represent carbazole groups.

(precoating layer); (2) L layers of $\mathrm{P}(\mathrm{C}-\mathrm{iB}) \mathrm{x}(\mathrm{L}=2-16, \mathrm{x}=5-15)$; (3) 4 layers of PVO (surface protection layers) (see Figure 1 (a)). The P(C-OD) LB film was prepared with the following sequence : (1) 2 layers of poly(octadecyl methacrylate) (PODMA); (2) L layers of P(C-OD) ( $L=1$ 4); (3) 4 layers of PVO (Figure 1 (b)). Hereafter, these films are called $i B x(L)$ and $O D(L)$. The P(C-iB) LB film with spacer layers has the following layer sequence : (1) 4 layers of PVO; (2) 2 layers of $\mathrm{P}(\mathrm{C}-\mathrm{iB}) 15$; (3) 2 layers of PVO (spacer layer); (4) 2 layers of $\mathrm{P}(\mathrm{C}-\mathrm{iB}) 15$; (5) 4 layers of PVO (Figure 1 (c)). The average distance $\left(R_{2 D}\right)$ between the carbazole chromophores in a 2dimensional plane was calculated from the composition of polymers and the surface area at the deposition. The data are shown in Table I.

Measurement. Steady state phosphorescence spectra were obtained with a Hitachi 850 spectrofluorophotometer fitted with a phosphorescence attachment. Phosphorescence decay curves were measured by a phosphorimeter assembled in our laboratory using a nitrogen laser as the excitation light source. The details of the system have been described elsewhere [2]. All spectroscopic measurements were performed at $77 \mathrm{~K}$.

\section{Results and discussion.}

Figure 2 (a) shows the phosphorescence spectra for iB5(L). All the samples exhibit almost 


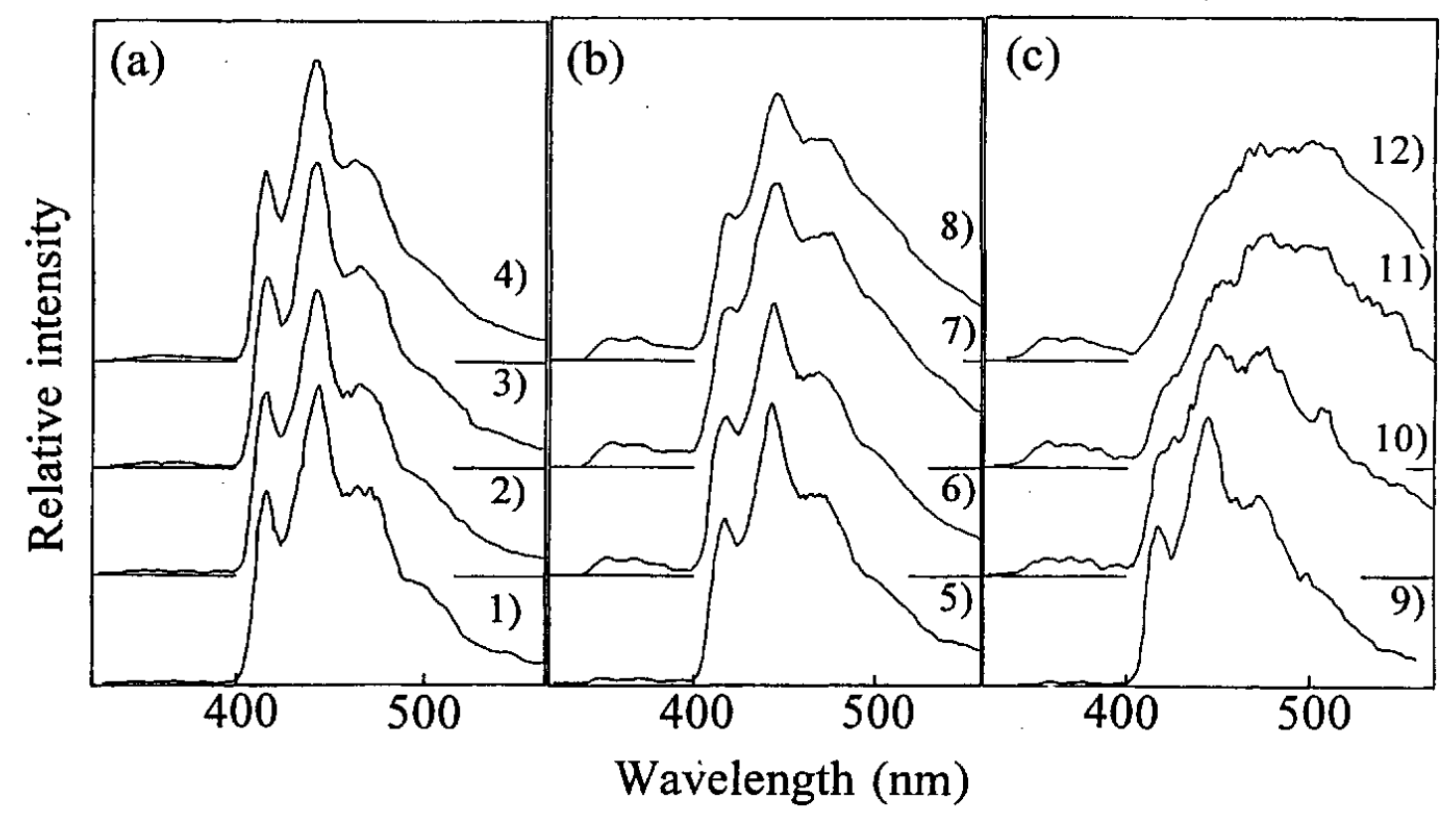

Fig. 2 Phosphorescence spectra for P(C-iB) LB films;

1) iB5(2), 2) iB5(4), 3) iB5(8), 4) iB5(16), 5) iB10(2), 6) iB10(4), 7) iB10(8), 8) iB10(16), 9) iB15(2), 10) iB15(4), 11) iB15(8), 12) iB15(16).

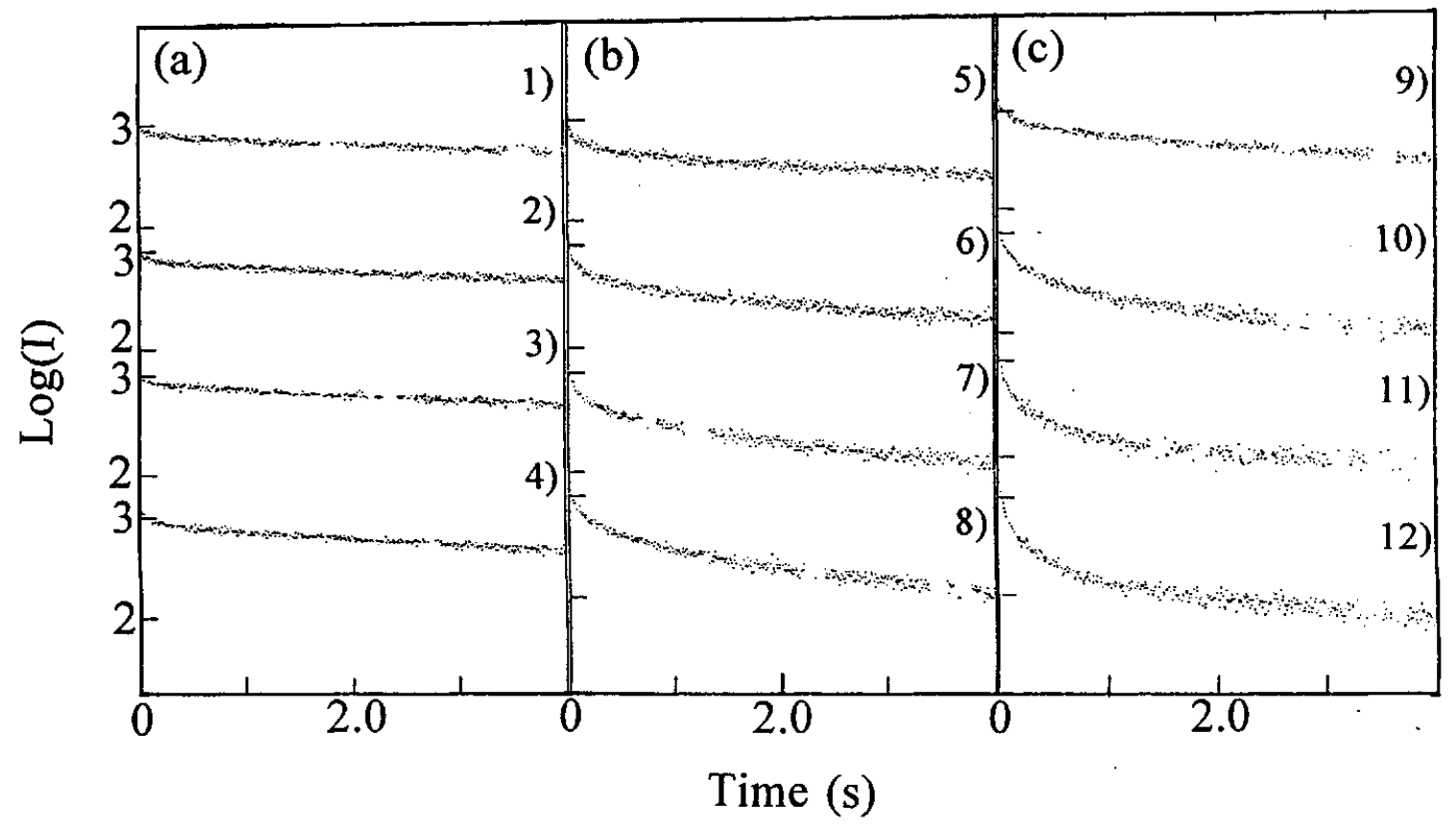

Fig. 3 Phosphorescence decay curves for $\mathrm{P}(\mathrm{C}-\mathrm{iB}) \mathrm{LB}$ films;

1) iB5(2), 2) iB5(4), 3) iB5(8), 4) iB5(16), 5) iBl0(2), 6) iBl0(4), 7) iBl0(8), 8) iB10(16), 9) iBl5(2), 10) iB15(4), 11) iB15(8), 12) iB15(16). 
the same emission spectra, consisting of the monomer phosphorescence of carbazole chromophore. This indicates that the triplet state chromophores in the $\mathrm{P}(\mathrm{C}-\mathrm{iB}) 5 \mathrm{LB}$ films have no obvious interaction with neighboring chromophores, and form no stabilized site such as a triplet excimer or a trap site. Figure 3(a) shows the phosphorescence decay curves for iB5(L). The phosphorescence decay curves are almost single exponential with a lifetime of 8-9 $\mathrm{s}$ for all samples. The lifetime is the same as that of an isolated carbazole chromophore in an amorphous film of P(CzEMA-co-MMA) [2]. In the iB5(L), triplet energy migration hardly occurs, since the $R_{2 D}(=1.90 \mathrm{~nm})$ is larger than the critical radius $\left(R_{0}\right)$ of the triplet energy transfer : $R_{0}$ between carbazole chromophores must be less than $1.5 \mathrm{~nm}$ [10-12].

Figures 2 (b) and 2 (c) show the phosphorescence spectra for $i B 10(L)$ and $i B 15(L)$. The spectrum for $\mathrm{L}=2$ and that for $\mathrm{L}=4$ are quite different. The former is identical to the monomer phosphorescence, but the latter is trap or excimer phosphorescence. The spectra for more layers than four are composed of trap phosphorescence for $\mathrm{iB} 10(\mathrm{~L})$ and excimer phosphorescence for iB15(L). Figures 3 (b) and 3 (c) show the phosphorescence decay curves for $\mathrm{iB} 10(\mathrm{~L})$ and $\mathrm{iB} 15(\mathrm{~L})$. All decay curves show a multi-exponential profile. As shown in Figure 2, delayed fluorescence emission observed around $350 \mathrm{~nm}$ is faint for $\mathrm{iB} 10(2)$ and $\mathrm{iB} 15(2)$, while it is clearly observed for

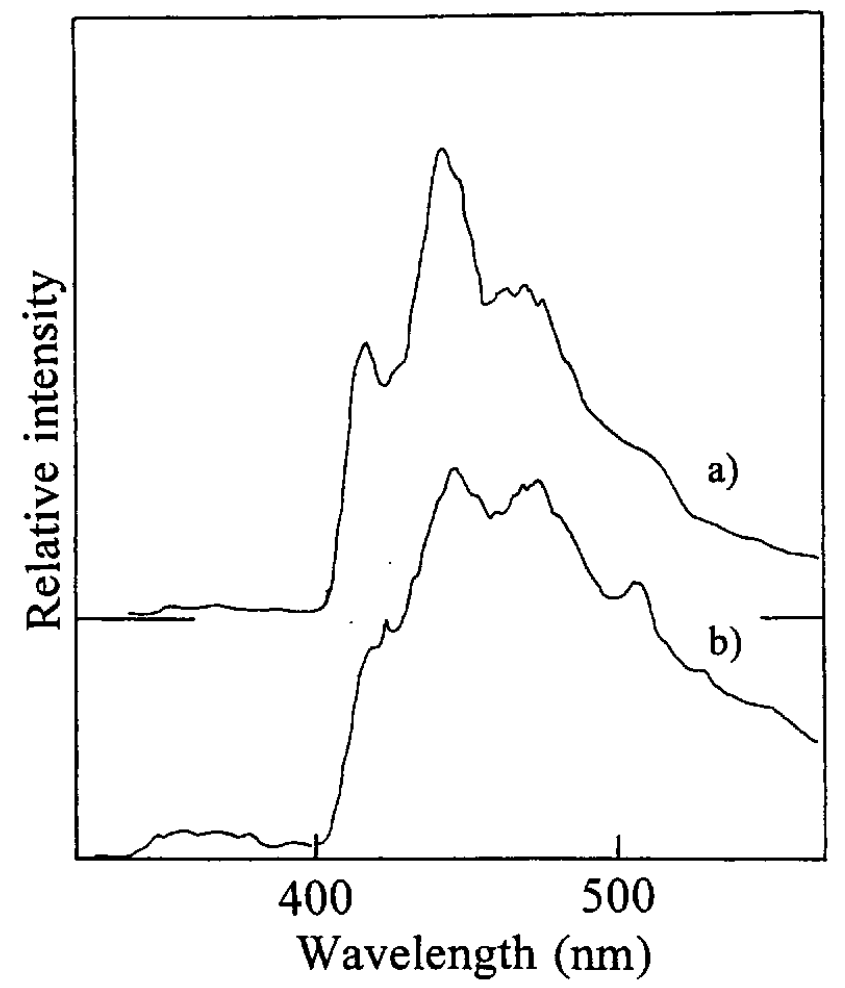

Fig. 4 Phosphorescence spectra for $\mathrm{P}(\mathrm{C}-\mathrm{iB}) 15 \mathrm{LB}$ films;

a) with PVO spacer and b) without spacer. 
$\mathrm{L}=4,8$ and 16. Therefore, the steep decays in the early stages for $\mathrm{L}=4,8$ and 16 are ascribed to the T-T annihilation. This indicates that the triplet energy migration in $\mathrm{L}=4,8$ and 16 occurs more vigorously than in $L=2$. These findings suggest that the average interchromophore distances for $L=4,8$ and 16 are shorter than that for $L=2$.

Figure 4 shows a comparison of the phosphorescence spectra for $\mathrm{P}(\mathrm{C}-\mathrm{iB}) 15$ with different layer sequences. The upper spectrum is the emission from the sample having spacer layers, and the lower is the emission without spacer layers, which is identical with that for iB15(4) in Figure 2 (c). Both samples have 4 layers of $\mathrm{P}(\mathrm{C}-\mathrm{iB}) 15$. However, the upper spectrum shows the monomeric emission, whereas the lower one shows the excimeric emission band. The sample separated with PVO layers is photophysically equivalent to iB15(2). These findings indicate the existence of interlayer interaction in $\mathrm{iB} 15(4)$, but not in $\mathrm{iB15}(2)$.

Since the $\mathrm{P}(\mathrm{C}$-iB) LB films were deposited as a Y-type film, we must consider a couple of

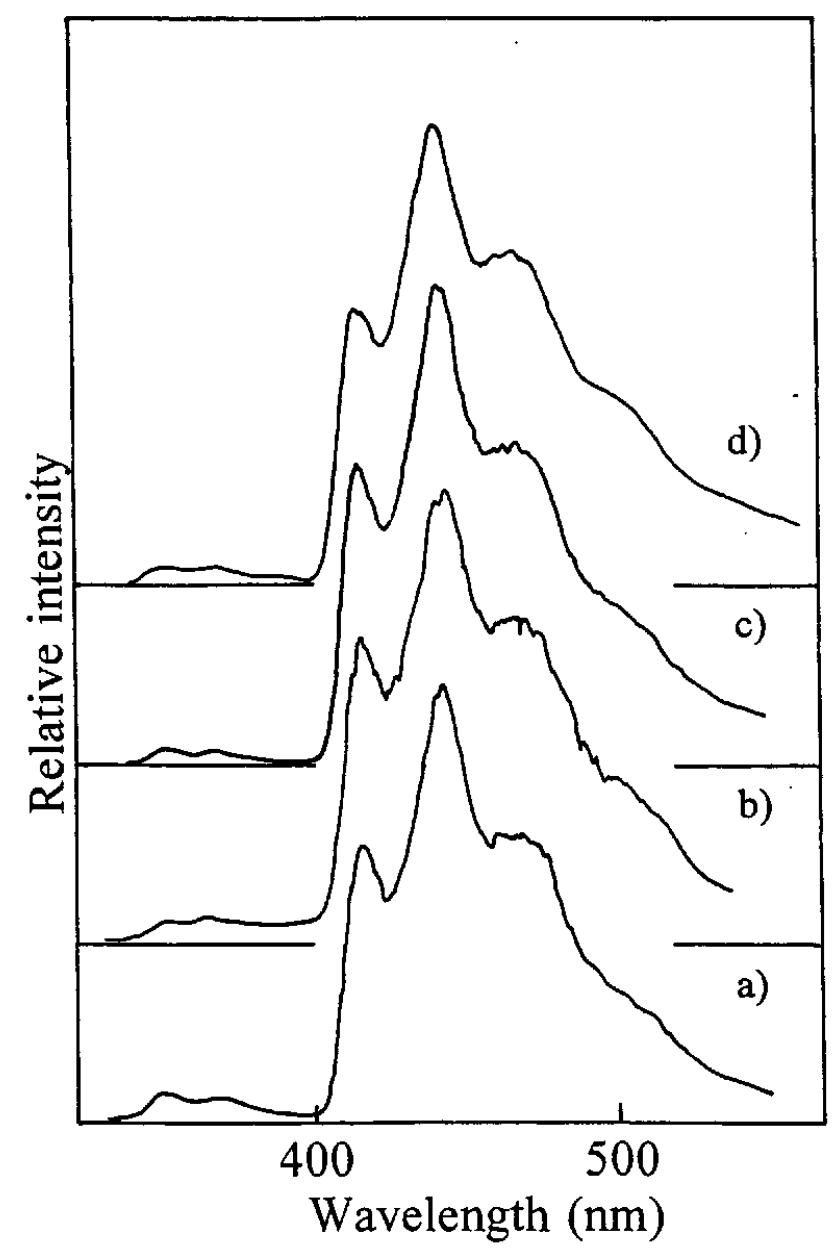

Fig. 5 Phosphorescence spectra for P(C-OD) LB films; a) $\mathrm{OD}(1)$, b) $\mathrm{OD}(2)$, c) $\mathrm{OD}(3)$, and d) $\mathrm{OD}(4)$. 
layers transferred with the downward and upward dips, as a structure unit. The inside of a couple of layers is hydrophilic and the outside is hydrophobic. The hydrophobic carbazole chromophore being attached to the polymer side chain should be localized at the hydrophobic surface of a pair of layers. If many layers are deposited subsequently, the chromophores easily come into contact with the next layer, even if each chromophore exists on a plane of LB films as shown in Figure 1 (a). Therefore, the plane density of chromophores at the hydrophobic interface between two adjacent layers would be twice of that for a monolayer. Under this assumption, the average interchromophore distance at the hydrophobic interface is calculated to be $1.05 \mathrm{~nm}$ for $\mathrm{P}(\mathrm{C}-\mathrm{iB}) 10$ and $0.93 \mathrm{~nm}$ for $\mathrm{P}(\mathrm{C}-\mathrm{iB}) \mathbf{1 5}$. These values are short enough to form a "shallow trap site" and a "triplet excimer", respectively. Figure 5 shows the phosphorescence spectra of OD(L) which has a very long side chain. These spectra are almost the same, and independent of the number of chromophoric layers, although the $R_{2 D}$ is nearly equal to that of $P(C-i B) 15$. This result supports the discussion mentioned above.

Cast films of $\mathrm{P}(\mathrm{CzEMA}-\mathrm{co}-\mathrm{MMA})$ showed excimer phosphorescence and multi-exponential phosphorescence decays, when the average interchromophore distance was shorter than $1.0 \mathrm{~nm}$ [2]. The decay profiles and the peak position of excimer phosphorescence are similar to those of $\mathrm{iB} 15(4), \mathrm{iB} 15(8)$, and $\mathrm{iB} 15(16)$, where the average interchromophore distances for these samples are around $1.0 \mathrm{~nm}$ as mentioned previously. The red-shifted spectra are also observed in a cast film with an average interchromophore distance of $1.2 \mathrm{~nm}$. We called this species "shallow trap site", which is formed by stabilization with a weak interaction between chromophores. The slightly red shifted spectra and multi-exponential decay profiles for $\mathrm{iB} 10(4), \mathrm{iB} 10(8), \mathrm{iB} 10(16), \mathrm{iB} 15(2)$ and $O D(L)$ are ascribed to the emission from "shallow trap site". It should be noted that the $R_{2 D}$ of $L B$ film to form a "shallow trap site" is $1.05-1.32 \mathrm{~nm}$ and the value is in good agreement with the distance for the cast film : $1.2 \mathrm{~nm}$. A similar relation in average distance was seen in the formation of triplet excimer. These findings suggest that the interchromophore distance is a good measure of triplet energy migration between triplet chromophores, whether the system is 2dimensional LB film or 3-dimensional amorphous polymer film.

The drastic change in phosphorescence spectrum at a particular distance must be due to the effect of energy migration, because the carbazole chromophore in LB film and in cast film could hardly change its conformation during the lifetime at $77 \mathrm{~K}$. Therefore the distance, 1.0-1.2 nm, indicates the critical distance at which the triplet begins to migrate vigorously in amorphous polymer matrices. 


\section{References}

1. (a) J. E. Guillet, Polymer Photophysics and Photochemistry, Cambridge University Press, Cambridge, U.K. (1985); (b) I. Soutar, Photophysical and Photochemical Tools in Polymer Science, M. A. Winnik, Ed., Dordrecht, The Netherlands (1986).

2. S. Ito, H. Katayama, and M. Yamamoto, Macromolecules, 21 (1988) 2456.

3. S. Ito, N. Numata, and M. Yamamoto, Macromolecules, 22 (1989) 2207.

4. H. Katayama, S. Ito, and M. Yamamoto, J. Photopolym. Sci. Technol., 4 (1991) 217.

5. H. Katayama, T. Tawa, S. Ito, and M. Yamamoto, J. Chem. Soc. Faraday Trans., 88 (1992) 2743.

6. H. Katayama, K. Hisada, M. Yanagida, S. Ohmori, S. Ito, and M. Yamamoto, Thin Solid Films, 224 (1993) 253.

7. S. Ito, S. Ohmori, and M. Yamamoto, Macromolecules, 25 (1992) 185.

8. S. Ohmori, S. Ito, and M. Yamamoto, Macromolecules, 24 (1991) 2377.

9. S. Ohmori, S. Ito, and M. Yamamoto, Macromolecules, 23 (1990) 4047.

10. V. L. Ermolaev, Sov. Phys. Dokl., 6 (1962) 600.

11. H. Katayama, S. Maruyama, S. Ito, Y. Tsujii, A. Tsuchida, and M. Yamamoto, J. Phys. Chem., 95 (1991) 3480.

12. H. Katayama, S. Ito, and M. Yamamoto, J. Phys. Chem., 96 (1992) 10115. 